\title{
From the Galileo Free Fall String to the Principle of Equivalence
}

\author{
Miroslav Pardy \\ Department of Physical Electronics \\ Masaryk University \\ Kotlářská 2, 61137 Brno, Czech Republic \\ e-mail:pamir@physics.muni.cz
}

September 17, 2021

\begin{abstract}
We consider the string with the length $l$, the left end and the right end of which is non relativistically accelerated by the constant acceleration $a$. We calculate the motion of such string and then the motion of the Galileo free fall string in gravity. The solutions are not identical. So, we distinguish between noninertial field and the gravity field and we discuss the principle of equivalence. In conclusion we suggest to drop charged objects from the very high tower Burj Khalifa in order to say crucial words on the principle of equivalence.
\end{abstract}

\section{Introduction}

It is well known that Galileo performed experiment in Pisa - later the famous experiment - with the result that the every falling body is falling with a uniform acceleration, the resistance of the medium being through which it was falling remained negligible. He also derived the correct kinematic law for the distance traveled during a uniform acceleration starting from rest, namely, that it is proportional to the square of the elapsed time. Prior to Galileo, Nicole Oresme, in the 14-th century, had derived the times-squared law for uniformly accelerated body, and Domingo de Soto had suggested in the 16-th century that bodies falling through a homogeneous medium would be uniformly accelerated. Soto, however, did not recognize the strictly uniform acceleration is only in a vacuum, and that it would otherwise eventually reach a uniform terminal velocity. Galileo expressed the time-squared law using geometrical constructions and mathematically precise words.

We here repeat the Galileo experiment in the generalized mathematical form. Namely with the string. We discuss the motion of the string accelerated by the Newton forces 
and by gravity and we discover substantial differences leading to the philosophy of the principle of equivalence.

\section{The uniformly accelerated string}

Let us consider the string with the length $l$, the left end of which is accelerated by the constant acceleration $a$ and the right end is accelerated also by the constant acceleration $a$.

Our problem is described by the wave equation (Koshlyakov, et al., 1962)

$$
u_{t t}=c^{2} u_{x x}+g(x, t)
$$

with the boundary conditions

$$
\begin{gathered}
u(x=0)=\kappa_{1}(t)=\frac{1}{2} a t^{2} \\
u(x=l)=\kappa_{2}(t)=\frac{1}{2} a t^{2}+l=\kappa_{1}(t)+l
\end{gathered}
$$

and with the initial conditions

$$
u(t=0)=f(x) ; \quad u_{t}(t=0)=F(x) .
$$

The problem cannot be solved by the standard Fourier method because the boundary conditions (2)-(3) are not homogenous. So, we introduce the auxiliary function (Koshlyakov et al., 1962)

$$
w(x, t)=\kappa_{1}(t)+\left[\kappa_{2}(t)-\kappa_{1}(t)\right] \frac{x}{l}
$$

with the boundary conditions

$$
w(x=0)=\kappa_{1}(t) ; \quad w(x=l)=\kappa_{1}(t)+l
$$

and the final solution we find in the form:

$$
u(x, t)=v(x, t)+w(x, t)
$$

with the boundary conditions

$$
v(x=0)=0 ; \quad v(x=l)=0
$$

and the initial conditions

$$
v(t=0)=f_{1}(x) ; \quad v_{t}(t=0)=F_{1}(x) .
$$

After insertion of $u=v+w$ into eq. (1), we get the following equation for $v$ and $w$ :

$$
v_{t t}=c^{2} v_{x x}+g(x, t)+c^{2} w_{x x}-w_{t t} .
$$

Then, if we use the definition of $w$ by eq. (5), we get equation for $v$ in the form:

$$
v_{t t}=c^{2} v_{x x}+g_{1}(x, t),
$$


where

$$
g_{1}(x, t)=g(x, t)-\kappa_{1}^{\prime \prime}(t)-\left[\kappa_{2}^{\prime \prime}(t)-\kappa_{1}^{\prime \prime}(t)\right] \frac{x}{l} .
$$

So, we see, that the last algebraic procedures lead to new system of equations. Namely:

$$
v_{t t}=c^{2} v_{x x}+g_{1}(x, t)
$$

with

$$
v(x=0)=0 ; \quad v(x=l)=0
$$

and

$$
v(t=0)=f_{1}(x) ; \quad v_{t}(t=0)=F_{1}(x) .
$$

It is easy to show that $g_{1}(x, t)=g-a$ and the system of equation to be solved is as follows:

$$
v_{t t}=c^{2} v_{x x}+g-a
$$

with

$$
v(x=0)=0 ; \quad v(x=l)=0
$$

and

$$
v(t=0)=f_{1}(x)=0 ; \quad v_{t}(t=0)=F_{1}(x)=0 .
$$

The solution of the system is well known (Koshlyakov et al., 1962) and so we write the final form:

$$
v(x, t)=\sum_{k=1}^{\infty} T_{k} \sin \left(\frac{k \pi x}{l}\right)
$$

where

$$
T_{k}(t)=\frac{2}{l \omega_{k}} \int_{0}^{t} d \tau \int_{0}^{l} G(\xi, \tau) \sin \omega_{k}(t-\tau) \sin \left(\frac{k \pi \xi}{l}\right) d \xi
$$

where

$$
\omega_{k}=\frac{k \pi c}{l} ; \quad G(\xi, \tau)=g-a
$$

\section{The Free fall of the string in gravity}

Let us consider the string with length $l$, the upper end is hanged in the gravity with the acceleration $g$ and the second end is free at time $t=0$. So the mathematical formulation of the problem is as follows (Koshlyakov, et al., 1962):

$$
u_{t t}=c^{2} u_{x x}+g
$$

with

$$
\begin{array}{ll}
u(x=0)=0 ; & u_{x}(x=l)=0 \\
u(t=0)=0 ; & u_{t}(t=0)=0 .
\end{array}
$$


Putting $u=v+w$, we get for $w$ the obligate system of equations:

$$
w_{t t}=c^{2} w_{x x}
$$

with the boundary conditions

$$
w(x=0)=0 ; \quad w_{x}(x=l)=0
$$

and the initial conditions

$$
w(t=0)=-v(t=0) ; \quad w_{t}(t=0)=-v_{t}(t=0) .
$$

It is possible to show (Koshlyakov, et al., 1962) that

$$
v=\frac{g x(2 l-x)}{2 c^{2}} .
$$

So, we can write

$$
f(x)=\frac{g x(x-2 l)}{2 c^{2}} ; \quad F(x)=0
$$

Then, by the standard method of integration, we get

$$
\begin{gathered}
u(x, t)=\frac{g x(2 l-x)}{2 c^{2}} \\
-\frac{16 g l^{2}}{\pi^{3} c^{2}} \sum_{k=1}^{\infty} \frac{1}{(2 k+1)^{3}} \cos \left(\frac{(2 k+1) \pi a t}{2 l}\right) \sin \left(\frac{(2 k+1) \pi x}{2 l}\right)
\end{gathered}
$$

and

$$
u(x=l)=\frac{g l^{2}}{2 c^{2}}-\frac{16 g l^{2}}{\pi^{3} c^{2}} \sum_{k=1}^{\infty} \frac{(-1)^{k}}{(2 k+1)^{3}} \cos \left(\frac{(2 k+1) \pi a t}{2 l}\right) .
$$

The maximal quantity $u_{\max }$ is at point $t=2 l / c$ and so we get

$$
u_{\text {max }}=\frac{g l^{2}}{2 c^{2}}+\frac{16 g l^{2}}{\pi^{3} c^{2}} \sum_{k=1}^{\infty} \frac{(-1)^{k}}{(2 k+1)^{3}}
$$

With regard to the mathematical formula

$$
\sum_{k=1}^{\infty} \frac{(-1)^{k}}{(2 k+1)^{3}}=\frac{\pi^{3}}{32}
$$

we get

$$
u_{\max }=\frac{g l^{2}}{c^{2}} .
$$

So, the length of the string (rod) is in the interval $\left(l, l+\frac{g l^{2}}{c^{2}}\right)$. 


\section{Discussion}

We have seen how to calculate the internal motion of the uniformly accelerated nonrelativistic of the length $l$ by the Newton force and by the gravity force which is the analogue of the experiment where Galileo dropped objects from the leaning tower of Pisa. Galileo have used two bodies made of the same material, differing only in size. He had in fact stated that, if the effects of air friction could be ignored, the two bodies would reach the ground at the same time. So, he supported the conclusion that the every falling body is falling with a uniform acceleration, the resistance of the medium being negligible. Galileo experimentation represented the kernel of scientific investigation and Galileo was keen to point this out (Frova et al., 2006).

Galileo experiment can be related to the Einstein equivalence principle with two reference frames, $\mathrm{K}$ and $\mathrm{K}^{\prime}$ where $\mathrm{K}$ is a uniform gravitational field, whereas $\mathrm{K}^{\prime}$ has no gravitational field but is uniformly accelerated in such a way that objects in the two frames experience identical forces. According to Einstein systems K and K' are physically exactly equivalent. This assumption of exact physical equivalence makes it impossible to speak of the absolute acceleration of the system of reference, just as the usual theory of relativity forbids to talk of the absolute velocity of a system. It makes the equal falling of all bodies in a gravitational field (Einstein, 1911).

Or, Inertia and gravity are identical; hence and from the results of special relativity theory it inevitably follows that the symmetric fundamental tensor $g_{\mu \nu}$ determines the metric properties of space, of the motion of bodies due to inertia in it, and, also, the influence of gravity (Einstein, 1918).

According to Fock (1964), principle of equivalence is understood to be the statement that in some sense a field of acceleration is equivalent to a gravitational field. It means that by introducing a suitable system of coordinates (which is usually interpreted as an accelerated frame of reference) one can so transform the equations of motion of a mass point in a gravitational field that in this new system they will have the appearance of equations of motion of a free mass point. Thus a gravitational field can, so to speak, be replaced, or rather imitated, by a field of acceleration. Owing to the equality of inertial and gravitational mass such a transformation is the same for any value of the mass of the particle. But it will succeed in its purpose only in an infinitesimal region of space, i.e. it will be strictly local. In the general case the transformation described corresponds mathematically to passing to a locally geodesic system of coordinates.

The principle of equivalence states that it is impossible to distinguish between the action on a particle of matter of a constant acceleration, or, of static support in a gravitational field (Lyle, 2008).

We have seen that the motion of the accelerated string by the non-gravity forces differs from the motion of the string caused by the gravity with the acceleration g.

The controversions between different opinions can be easily solved with regard to the physical definition of gravity and inertia. Namely: gravity is form of matter in the physical vacuum. And inertia is the result of the interaction of the massive body with quantum vacuum being the physical medium.

It is well known that synchrotron radiation influences the motion of the electron in accelerators. The corresponding equation which describes the classical motion is so called the Lorentz-Dirac equation, which differs from the the so called Lorentz equation

$$
m c \frac{d u_{\mu}}{d s}=\frac{e}{c} F_{\mu \nu} u^{\nu}
$$


only by the additional term which describes the radiative corrections. So, the equation with the radiative term is as follows (Landau et al., 1988):

$$
m c \frac{d u_{\mu}}{d s}=\frac{e}{c} F_{\mu \nu} u^{\nu}+g_{\mu},
$$

where $u_{\mu}$ is the four-velocity and the radiative term was derived by Landau et al. in the form (Landau et al., 1988):

$$
g_{\mu}=\frac{2 e^{3}}{3 m c^{3}} \frac{\partial F_{\mu \nu}}{\partial x^{\alpha}} u^{\nu} u^{\alpha}-\frac{2 e^{4}}{3 m^{2} c^{5}} F_{\mu \alpha} F^{\beta \alpha} u_{\beta}+\frac{2 e^{4}}{3 m^{2} c^{5}}\left(F_{\alpha \beta} u^{\beta}\right)\left(F^{\alpha \gamma} u_{\gamma}\right) u_{\mu} .
$$

The last equation can be easily converted into equation for charged particle moving in gravity. However, the term describing the radiation caused by gravity is not present (Landau, et al., 1988).

It was proved by author (Pardy, 2009) that synchrotron radiation influences the spin motion of the electron in accelerators. The corresponding equation which describes the classical spin motion is so called the Bargman-Michel-Telegdi-Pardy and is of the form (Pardy, 2009):

$$
\begin{gathered}
\frac{d a_{\mu}}{d s}=2 \mu F_{\mu \nu} a^{\nu}-2 \mu^{\prime} u_{\mu} F^{\nu \lambda} u_{\nu} a_{\lambda}+ \\
\Lambda u_{\mu}\left\{\frac{2 e^{3}}{3 m c^{3}} \frac{\partial F_{\lambda \nu}}{\partial x^{\alpha}} u^{\nu} u^{\alpha}-\right. \\
\left.\frac{2 e^{4}}{3 m^{2} c^{5}} F_{\lambda \alpha} F^{\beta \alpha} u_{\beta}+\frac{2 e^{4}}{3 m^{2} c^{5}}\left(F_{\alpha \beta} u^{\beta}\right)\left(F^{\alpha \gamma} u_{\gamma}\right) u_{\lambda}\right\} a^{\lambda} .
\end{gathered}
$$

Let us remark that the conversion of this equation to the situation where the interaction with the gravitational field is present, was not still derived.

Let us remark, that free fall of the positronium is of the same law as the free fall of an electron, or, positron apart. Also, free fall of the protonium is of the same law as the free fall of the proton, or, antiproton apart. It was experimentally verified. It means that the charge interaction with gravity is zero. Gravity interact only with mass and the result of such interaction is the free fall with emission of gravitons. In case of the binary system it was confirmed by NASA and the spectral formula of the emission of gravitons by the binary was calculated by author (Pardy, 1983; 1994a; 1994b; 2011; 2018; 2019). In case of the existence of the gravitational index of refraction, the gravitational Cherenkov radiation is possible (Pardy, 1994c; 1994d).

While Galileo dropped objects from the leaning tower of Pisa, now, we have possibility to drop charged objects from the very high tower Burj Khalifa, in order to confirm the law that charged objects accelerated by the gravitational field do not radiate the electromagnetic energy. It is not excluded that such experiment with the adequate title Galileo-Pardy-Burj Khalifa project will be realized sooner, or, later. The project is cheaper than LHC.

\section{References}

Einstein, A. (1911). Über den Einflus der Schwerkraft auf die Ausbreitung des Lichtes, Ann. Phys. 1911, 35, 898-908. ibid. On the Influence of Gravitation on the Propagation of Light, Vol. 3, Doc. 23, 485-497 (Einstein 1911h). 
Einstein, A. (1918). Principieless zur allgemeinen Relativitätstheorie, Ann. Phys. 1918, 55, 241-244. ibid. On the Foundations of the General Theory of Relativity Vol. 7, Doc. 4, 37-44 (Einstein 1918e).

Fock, V. Theory of space, time and and gravitation, Second revised ed., (Pergamon Press, Oxford, London, ... 1964).

Frova, A. and Marenzana, M. Thus Spoke Galileo, The Great Scientist's Ideas and Their Relevance to the Present Day, (Oxford University Press, 2006).

Koshlyakov, N. S., Gliner, E. V. and M. M. Smirnov, The Differential Equations of Mathematical Physics. (Nauka, Moscow, 1962). (in Russian).

Landau, L. D. and Lifshitz, E. M. , The Classical Theory of Fields, Fourth Revised English Edition, (Amsterdam, Boston, Heidelberg, London, ..., 1987).

Lyle, S. N. Uniformly Accelerating Charged Particles, A Threat to the Equivalence Principle, (Springer-Verlag, Berlin, Heidelberg, 2008).

Pardy, M. (2009), The bremsstrahlung equation for the spin motion in electromagnetic field, International Journal of Theoretical Physics, Volume 48, Number 11, pp. 3241-3248; arXiv: hep-ph/0809.3888 v1, acc-ph/0801.2483.

Pardy, M. (1983). The synchrotron production of gravitons by the binary system, GRG 15, No. 11 (1983), 1027.

Pardy, M. (1994a). The High-energy gravitons from the binary: Preprint: CERN-TH. $7239 / 94$.

Pardy, M. (1994b). The quantum energy loss of a binary system: CERN-TH. 7299/94.

Pardy, M. (1994c). The gravitational Cerenkov radiation with radiative corrections: CERN-TH.7270/94.

Pardy, M. (1994d). The gravitational Čerenkov radiation with radiative corrections: Phys. Lett. B 336, 362.

Pardy, M. (2011). The radiation of the gravitational and electromagnetic binary pulsars; pp. 97-123. in: Pulsars: Discoveries, Functions and Formation (Editors: Peter A. Travelle, Nova Science Publishers, Series: Space Science).

Pardy, M. (2018). The black hole binary gravitons and thermodynamics, viXra: $1810.0304 \mathrm{v} 1$.

Pardy, M. (2019). The Black Hole Binary Gravitons and Related Problems, IntechOpen. Book title: New Ideas Concerning Black Holes and the Evolution of the Universe 